\title{
QUASICONFORMAL HOMEOMORPHISMS BETWEEN RIEMANN SURFACES
}

\author{
CABIRIA ANDREIAN CAZACU \\ Faculty of Mathematics, University of Bucharest \\ Bucureşti, Romania \\ VICTORIA STANCIU \\ Department of Mathematics, Polytechnical Institute of Bucharest \\ Bucureşti, Romania
}

0. Introduction. In his lectures "Characteristic properties of quasidisks" [8], F. W. Gehring proved the following.

Theorem. Let $f: \widehat{\mathbb{C}} \rightarrow \widehat{\mathbb{C}}$ be a $K$-qc (K-quasiconformal $)$ homeomorphism with $f(\infty)=\infty$. If $z_{j}, j=0,1,2$, are three distinct points in $\mathbb{C}$ and $z_{j}^{\prime}=f\left(z_{j}\right)$, then

$$
\left|z_{1}-z_{0}\right| \leq\left|z_{2}-z_{0}\right| \quad \text { implies } \quad\left|z_{1}^{\prime}-z_{0}^{\prime}\right| \leq e^{8 K}\left|z_{2}^{\prime}-z_{0}^{\prime}\right|
$$

Our initial aim was to extend this theorem to $K$-qc homeomorphisms between Riemann surfaces. It was an immediate observation that Gehring's theorem may be rewritten by means of Evans-Selberg potential or the Sario capacity function and we shall use the latter to cover both the parabolic and the hyperbolic cases. Indeed, the Sario capacity function of $\mathbb{C}$ with respect to $z_{0}$ is $p_{\mathbb{C}}\left(z, z_{0}\right)=\log \left|z-z_{0}\right|$. On the other hand, $f$ may be interpreted by restriction as a $K$-qc homeomorphism $f \mid \mathbb{C}: \mathbb{C} \rightarrow \mathbb{C}$, so that if we denote $p_{\mathbb{C}}\left(z, z_{0}\right)$ by $\tau$ and $p_{\mathbb{C}}\left(z^{\prime}, z_{0}^{\prime}\right)$ by $\tau^{\prime}$, Gehring's theorem takes the form:

Let $f: \mathbb{C} \rightarrow \mathbb{C}$ be a $K$-qc homeomorphism, $z_{j}, j=0,1,2$, distinct points in $\mathbb{C}$, $z_{j}^{\prime}=f\left(z_{j}\right), \tau_{k}=p_{\mathbb{C}}\left(z_{k}, z_{0}\right)$ and $\tau_{k}^{\prime}=p_{\mathbb{C}}\left(z_{k}^{\prime}, z_{0}^{\prime}\right), k=1,2$. Then

$$
\tau_{1} \leq \tau_{2} \quad \text { implies } \quad \tau_{1}^{\prime} \leq \tau_{2}^{\prime}+8 K
$$

1991 Mathematics Subject Classification: 30C62, 30F15.

The paper is in final form and no version of it will be published elsewhere. 
In [4] we succeeded in extending this theorem to the class $R_{p}$ of Riemann surfaces. The proof was based on a theorem of normal families [5], I, and on results about the behaviour of the level lines of the capacity function under $K$-qc homeomorphisms [6], which led us further to other distortion problems [7]. The present paper is a renewed synthesis of this research.

\section{Compactness of families of $K$-qc homeomorphisms between Rie- mann surfaces}

1.1. In what follows we consider Riemann surfaces endowed with the metric corresponding to the conformal type of their universal coverings and the l.u. (locally uniform, [11], p. 14) convergence with respect to this metric. Points on Riemann surfaces and local parameters will be denoted by the same letters, e.g. $z, z^{\prime}$.

THEOREM 1.0. Let $R$ and $R^{\prime}$ be Riemann surfaces not conformally equivalent to $\mathbb{C}$ and $\widehat{\mathbb{C}}, M$ and $M^{\prime}$ compact subsets of $R$ and $R^{\prime}$ respectively, and $\mathcal{F}$ the family of $K$-qc homeomorphisms $f: R \rightarrow R^{\prime}$ for which

$$
f(M) \subset M^{\prime} .
$$

If $\mathcal{F} \neq \emptyset$, then $\mathcal{F}$ is normal and closed. The same result holds if condition (1.1) is replaced by

$$
f(M)=M^{\prime} .
$$

As in [5], I and II, we prove this theorem in two steps: first we lift the family $\mathcal{F}$ to the universal coverings $(\widehat{R}, \pi, R)$ and $\left(\widehat{R}^{\prime}, \pi^{\prime}, R^{\prime}\right)$ with a convenient normalization and show in Theorem 1.1 that the lifted family $\widehat{\mathcal{F}}$ is normal and closed, and then we deduce in Theorem 1.2 from these properties of $\widehat{\mathcal{F}}$ the same properties for $\mathcal{F}$.

1.2. In order to establish these theorems we use the following propositions.

Proposition 1.1. Let $\left\{f_{n}\right\}, n \in \mathbb{N}^{*}$, be a sequence of homeomorphisms between Riemann sufraces, $f_{n}: R \rightarrow R^{\prime}$, and for each $n$, let $\widehat{f}_{n}: \widehat{R} \rightarrow \widehat{R}^{\prime}$ be a lifting of $f_{n}$ with respect to the universal coverings $(\widehat{R}, \pi, R)$ and $\left(\widehat{R}^{\prime}, \pi^{\prime}, R^{\prime}\right)$ such that the sequence $\left\{\widehat{f}_{n}\right\}$ converges l.u. to a homeomorphism $\widehat{f}_{0}: \widehat{R} \rightarrow \widehat{R}^{\prime}$. Then

(i) $\widehat{f}_{0}$ is the lifting of a homeomorphism $f_{0}: R \rightarrow R^{\prime}$ and

(ii) the sequence $\left\{f_{n}\right\}$ converges l.u. to $f_{0}$.

Proof. (i) Consider the covering groups $G$ and $G^{\prime}$ of $(\widehat{R}, \pi, R)$ and $\left(\widehat{R}^{\prime}, \pi^{\prime}, R^{\prime}\right)$ respectively. To prove the existence of the homeomorphism $f_{0}$ it is necessary and sufficient to verify that for each $T \in G$ there exists $T^{\prime} \in G^{\prime}$ such that $\widehat{f}_{0} T \widehat{f}_{0}^{-1}=T^{\prime}$ ([11], p. 145). For every index $n$, there exists $T_{n}^{\prime} \in G^{\prime}$ with $\widehat{f}_{n} T \widehat{f}_{n}^{-1}=T_{n}^{\prime}$. Let $\widehat{z}^{\prime}$ be an arbitrary but fixed point in $\widehat{R}^{\prime}$. The sequence $\left\{T_{n}^{\prime} \widehat{z}^{\prime}\right\}$, being convergent and contained in the discrete orbit $G^{\prime} \widehat{z}^{\prime}$, must be stationary and, as $G^{\prime}$ is a fixed point 
free group, $T_{n}^{\prime}=T^{\prime}$ for sufficiently large $n$ and a certain $T^{\prime} \in G^{\prime}$. By passing to the limit in $\widehat{f}_{n} T \widehat{f}_{n}^{-1}=T^{\prime}$ one obtains $\widehat{f}_{0} T \widehat{f}_{0}^{-1}=T^{\prime}$.

(ii) follows easily from the l.u. convergence of $\left\{\widehat{f}_{n}\right\}$ and the properties of the metric: $d\left(f_{n}(\xi), f_{0}(\xi)\right) \leq d\left(\widehat{f}_{n}(\widehat{\xi}), \widehat{f}_{0}(\widehat{\xi})\right)$, for any $\xi \in R, \widehat{\xi} \in \pi^{-1}(\xi)$, where $d$ denotes the distance in the metric on $R$ and $\widehat{R}$ respectively.

Proposition 1.2. If in Proposition 1.1 the $f_{n}$ are $K-q c$, then so are $\widehat{f}_{n}, \widehat{f}_{0}$ and $f_{0}$.

1.3. TheOREM 1.1. Under the hypotheses of Theorem 0.1 and with the above notations fix arbitrary points $z_{0} \in M$ and $\widehat{z}_{0} \in \pi^{-1}\left(z_{0}\right)$, and a compact set $\widehat{M}^{\prime}$ with $\pi^{\prime}\left(\widehat{M}^{\prime}\right) \supset M^{\prime}$. The family $\widehat{\mathcal{F}}$ of all homeomorphisms $\widehat{f}: \widehat{R} \rightarrow \widehat{R}^{\prime}$ such that $\widehat{f}$ is the lifting of a homeomorphism $f \in \mathcal{F}$ normalized by the condition $\widehat{f}\left(\widehat{z}_{0}\right) \in \widehat{M}^{\prime}$, is normal and closed.

Proof for both cases (1.1) and (1.2). The hyperbolic case: $\widehat{R}=\widehat{R}^{\prime}=D$ the unit disc. The family $\widehat{\mathcal{F}}$ is normal by Theorem 5.1.1 of [12], since each $\widehat{f} \in \widehat{\mathcal{F}}$ omits $\widehat{\mathbb{C}} \backslash D$.

Consider a l.u. convergent sequence $\left\{\widehat{f}_{n}\right\} \subset \widehat{\mathcal{F}}, n \in \mathbb{N}^{*}$. According to Theorem 5.5 of [12], $\widehat{f}_{0}=\lim _{n \rightarrow \infty} \widehat{f}_{n}$ is a $K$-qc homeomorphism $D \rightarrow D$, for $\widehat{f}_{0}\left(\widehat{z}_{0}\right) \in \widehat{M}^{\prime} \subset D$. By Propositions 1.1 and 1.2 it follows that $\widehat{f}_{0}$ is the lifting of a $K$-qc homeomorphism $f_{0}: R \rightarrow R^{\prime}$ and if $f_{n} \in \mathcal{F}$ corresponds to $\widehat{f}_{n}$, the sequence $\left\{f_{n}\right\}$ l.u. converges to $f_{0}$. Then one easily sees that $f_{0} \in \mathcal{F}$, therefore $\widehat{f_{0}} \in \widehat{\mathcal{F}}$.

Case of the torus: $R=\mathbb{C} / \mathbb{Z} \omega_{1}+\mathbb{Z} \omega_{2}$ and $R^{\prime}=\mathbb{C} / \mathbb{Z} \omega_{1}^{\prime}+\mathbb{Z} \omega_{2}^{\prime}, \omega_{j}, \omega_{j}^{\prime} \in \mathbb{C}^{*}$, $j=1,2, \operatorname{Im}\left(\omega_{2} / \omega_{1}\right)>0$ and $\operatorname{Im}\left(\omega_{2}^{\prime} / \omega_{1}^{\prime}\right)>0, \widehat{R}=\widehat{R}^{\prime}=\mathbb{C}$.

If $f: R \rightarrow R^{\prime}$ is a homeomorphism and $\widehat{f}: \mathbb{C} \rightarrow \mathbb{C}$ a lifting of $f$, then

$$
\widehat{f}\left(\widehat{z}+m \omega_{1}+n \omega_{2}\right)=\widehat{f}(\widehat{z})+m \widehat{\omega}_{1}^{\prime}+n \widehat{\omega}_{2}^{\prime},
$$

where $\widehat{\omega}_{j}^{\prime}=\widehat{f}\left(\widehat{\xi}+\omega_{j}\right), \widehat{\xi}=\widehat{f}^{-1}(0)$ and $m, n \in \mathbb{Z}$.

Let $f$ be $K$-qc. Take the parallelogram $P$ with vertices $\widehat{z}_{0}, \widehat{z}_{0}+\omega_{1}, \widehat{z}_{0}+\omega_{1}+\omega_{2}$, $\widehat{z}_{0}+\omega_{2}$ and denote by $C_{j}$ the family of segments in $P$ which are parallel to the side $\widehat{z}_{0}, \widehat{z}_{0}+\omega_{j}$, by $P^{\prime}$ the image $\widehat{f}(P)$ and by $A^{\prime}$ the area of the parallelogram with vertices $0, \omega_{1}^{\prime}, \omega_{1}^{\prime}+\omega_{2}^{\prime}, \omega_{2}^{\prime}$, which is equal to the area of $P^{\prime}$, since $\widehat{\omega}_{j}^{\prime}=m_{j}^{\prime} \omega_{1}^{\prime}+n_{j}^{\prime} \omega_{2}^{\prime}$ and $m_{1}^{\prime} n_{2}^{\prime}-m_{2}^{\prime} n_{1}^{\prime}=1$. It follows that $\left|\widehat{\omega}_{j}^{\prime}\right|^{2} \leq K A^{\prime} / \operatorname{Mod} C_{j}$, i.e. we have obtained an upper bound for $\left|\widehat{\omega}_{j}^{\prime}\right|$, say $\varrho$, depending only on $R, R^{\prime}$ and $K$.

The family $\widehat{\mathcal{F}}$ in Theorem 1.1 is normal by Theorem 5.1.2 of [12] since every $\widehat{f} \in \widehat{\mathcal{F}}$ omits $\infty$ and $\widehat{f}\left(\widehat{z}_{0}+\omega_{j}\right)=\widehat{z}_{0}^{\prime}+\widehat{\omega}_{j}^{\prime}$ with $\widehat{z}_{0}^{\prime}=\widehat{f}\left(\widehat{z}_{0}\right) \in \widehat{M}^{\prime}$ and $\widehat{\omega}_{j}^{\prime} \in \operatorname{cl} D(0, \varrho)$.

Consider again a l.u. convergent sequence $\left\{\widehat{f}_{k}\right\} \subset \widehat{\mathcal{F}}, k \in \mathbb{N}^{*}$. According to Theorems 5.2 and 5.3 in [12], $\widehat{f}_{0}=\lim _{k \rightarrow \infty} \widehat{f}_{k}$ is a $K$-qc homeomorphism $\mathbb{C} \rightarrow \mathbb{C}$, since it takes an infinity of values. Indeed, set as before $\widehat{\xi}_{k}=\widehat{f}_{k}^{-1}(0)$ 
and $\widehat{\omega}_{j k}^{\prime}=\widehat{f}_{k}\left(\widehat{\xi}_{k}+\omega_{j}\right)$; clearly $\widehat{\omega}_{j k}^{\prime} \neq 0$ and $\widehat{\omega}_{j k}^{\prime} \in \mathbb{Z} \omega_{1}^{\prime}+\mathbb{Z} \omega_{2}^{\prime}$. By passing to the limit one obtains from the relation $\widehat{f}_{k}\left(\widehat{z}+\omega_{j}\right)=\widehat{f}_{k}(\widehat{z})+\widehat{\omega}_{j k}^{\prime}$ the existence of $\widehat{\omega}_{j 0}^{\prime}=\lim _{k \rightarrow \infty} \widehat{\omega}_{j k}^{\prime} \neq 0$, and from (1.3) written for $\widehat{f}_{k}$ the equality $\widehat{f}_{0}\left(\widehat{z}_{0}+m \omega_{1}+\right.$ $\left.n \omega_{2}\right)=\widehat{f}_{0}\left(\widehat{z}_{0}\right)+m \widehat{\omega}_{10}^{\prime}+n \widehat{\omega}_{20}^{\prime}$.

The closedness of $\widehat{\mathcal{F}}$ follows as in the hyperbolic case.

Case of $R=R^{\prime}=\mathbb{C}^{*}$. One proceeds as for the torus (by using the universal covering $\left(\mathbb{C}, \exp , \mathbb{C}^{*}\right)$ one obtains instead of $(1.3)$ the relation $\widehat{f}(\widehat{z}+2 \pi n i)=$ $\widehat{f}(\widehat{z}) \pm 2 \pi n i)$ or even directly as in [5], I.

\subsection{Propositions 1.1 and 1.2 imply}

THEOREM 1.2. Let $\mathcal{F}$ be a family of homeomorphisms (in particular of $K-q c$ homeomorphisms) $f: R \rightarrow R^{\prime}$ between two Riemann surfaces $R$ and $R^{\prime}$, and $\widehat{\mathcal{F}}$ a family consisting of at least a lifting $\widehat{f}: \widehat{R} \rightarrow \widehat{R}^{\prime}$ with respect to the universal coverings $(\widehat{R}, \pi, R)$ and $\left(\widehat{R}^{\prime}, \pi^{\prime}, R^{\prime}\right)$ for every $f \in \mathcal{F}$. If $\widehat{\mathcal{F}}$ is normal and closed, then $\mathcal{F}$ is also normal and closed.

1.5. R e mark 1.1. A special case of Theorem 1.0 and 1.1 is given by $M=\left\{z_{0}\right\}$ and $M^{\prime}=\left\{z_{0}^{\prime}\right\}$ (see [5], I). Of course sometimes it is useful to consider the case $M=\left\{z_{0}\right\}, M^{\prime}$ an arbitrary compact in $R^{\prime}$ (see [5], II).

Remark 1.2. Case $R=R^{\prime}=\mathbb{C}$. If $M=\left\{z_{0}\right\}$, the family $\mathcal{F}$ is not normal as the example $\left\{n\left(z-z_{0}\right)\right\}, n \in \mathbb{N}^{*}$, shows. However, if $M$ contains at least two distinct points, then $\mathcal{F}$ is normal and closed in the case (1.2), and normal but not closed in the case (1.1) as follows from the example $\{z / n\}, n \in \mathbb{N}^{*}, M=M^{\prime}=\bar{D}$ (see [5], II).

2. Distortion of the level lines of some principal functions under $K$-qc homeomorphisms

2.1. Let $R$ be an open Riemann surface, $\Gamma$ its ideal boundary, $M$ a compact subset in $R$ upon which we shall set other conditions in particular cases.

We shall deal with a harmonic function $t: R \backslash M \rightarrow\left(\tau_{0}, \mathcal{T}_{0}\right) \subset \mathbb{R}$ tending to constant limits $\tau_{0}$ as $z \in R \backslash M$ tends to $\partial M$ and $\mathcal{T}_{0}$ as $z \rightarrow \Gamma, \tau_{0}, \mathcal{T}_{0} \in[-\infty,+\infty]$, with the properties:

(i) The level lines $c_{\tau}:=\{z \in R \backslash M: t(z)=\tau\}$ are compact in $R \backslash M$ and they define regular exhaustions of $R$ by means of $\Pi_{\tau}:=M \cup\{z \in R \backslash M: t(z)<\tau\}$, $\tau \in\left(\tau_{0}, \mathcal{T}_{0}\right)$.

(ii) $\int_{c_{\tau}} * d t=a$ for some constant $a>0$, such that if $\tau_{1}<\tau_{2}, \tau_{j} \in\left(\tau_{0}, \mathcal{T}_{0}\right)$, the family of level lines $c_{\tau_{1} \tau_{2}}:=\left\{c_{\tau}: \tau \in\left[\tau_{1}, \tau_{2}\right]\right\}$ is an extremal family for the modulus of $\Pi_{\tau_{1} \tau_{2}}:=\operatorname{cl}\left(\Pi_{\tau_{2}} \backslash \Pi_{\tau_{1}}\right)$ with respect to its boundary partition given by $c_{\tau_{1}}$ and $c_{\tau_{2}}$. Namely for this modulus, denoted by $\operatorname{Mod} \Pi_{\tau_{1} \tau_{2}}$, which is equal by definition to the modulus of the family of curves in $\Pi_{\tau_{1} \tau_{2}}$ separating $c_{\tau_{1}}$ from 
$c_{\tau_{2}}$, we have (see $\left.[2]\right)$

$$
\operatorname{Mod} \Pi_{\tau_{1} \tau_{2}}=\operatorname{Mod} c_{\tau_{1} \tau_{2}}=\left(\tau_{2}-\tau_{1}\right) / a .
$$

Different functions $t$ are characterized by adding other conditions (as in the examples discussed below in 2.4) but only these general ones are involved in our results.

On the other hand, the existence of a function $t$ depends on $R$ and $M$ (a more precise notation for $t$ should be e.g. $t_{R}(, M)$ ), and we shall denote by $R_{t}$ the class of those open Riemann surfaces on which such a function $t$ exists.

Setting of the Problem. Let $R$ and $R^{\prime}$ be Riemann surfaces of class $R_{t}, \Gamma$ and $\Gamma^{\prime}$ their ideal boundaries, $M$ and $M^{\prime}$ compact sets in $R$ and $R^{\prime}$ respectively, such that there are functions $t=t_{R}(, M)$ and $t^{\prime}=t_{R^{\prime}}\left(, M^{\prime}\right)$. Suppose that $f$ : $R \rightarrow R^{\prime}$ is a $K$-qc homeomorphism with $f(M)=M^{\prime}$. We ask about a connection between $f c_{\tau}$ and the level lines $c_{\tau^{\prime}}^{\prime}=\left\{z^{\prime} \in R^{\prime} \backslash M^{\prime}: t^{\prime}\left(z^{\prime}\right)=\tau^{\prime}\right\}$.

In what follows we introduce for $t^{\prime}$ on $R^{\prime}$ the notations $c_{\tau_{1}^{\prime} \tau_{2}^{\prime}}^{\prime}, \Pi_{\tau^{\prime}}^{\prime}$ and $\Pi_{\tau_{1}^{\prime} \tau_{2}^{\prime}}^{\prime}$ similar to those for $t$ on $R$.

2.2. First we shall consider a single homeomorphism $f$ and define two auxiliary functions

and

$$
\tau_{0}^{\prime}(\tau, f)=\min \left\{\tau^{\prime}=t^{\prime}\left(z^{\prime}\right): z^{\prime} \in f c_{\tau}\right\}
$$

$$
\mathcal{T}_{0}^{\prime}(\tau, f)=\max \left\{\tau^{\prime}=t^{\prime}\left(z^{\prime}\right): z^{\prime} \in f c_{\tau}\right\} .
$$

Thus $f c_{\tau}$ is included in $\Pi_{\tau_{0}^{\prime}(\tau, f) \mathcal{T}_{0}^{\prime}(\tau, f)}^{\prime}$ or reduces to $c_{\tau^{\prime}}^{\prime}$ when

$$
\tau^{\prime}=\tau_{0}^{\prime}(\tau, f)=\mathcal{T}_{0}^{\prime}(\tau, f)
$$

In the first case the distortion of $f c_{\tau}$ from the level lines $c_{\tau^{\prime}}^{\prime}$ could be measured by

$$
\operatorname{Mod} c_{\tau_{0}^{\prime}(\tau, f) \mathcal{T}_{0}^{\prime}(\tau, f)}^{\prime}=\left(\mathcal{T}_{0}^{\prime}(\tau, f)-\tau_{0}^{\prime}(\tau, f)\right) / a .
$$

THEOREM 2.1. The functions $\tau_{0}^{\prime}(\tau, f)$ and $\mathcal{T}_{0}^{\prime}(\tau, f)$ are strictly increasing in $\tau$, and if $\tau_{1} \leq \tau_{2}$ then

$$
K^{-1}\left[\tau_{0}^{\prime}\left(\tau_{2}, f\right)-\mathcal{T}_{0}^{\prime}\left(\tau_{1}, f\right)\right] \leq \tau_{2}-\tau_{1} \leq K\left[\mathcal{T}_{0}^{\prime}\left(\tau_{2}, f\right)-\tau_{0}^{\prime}\left(\tau_{1}, f\right)\right] .
$$

Proof. $\mathcal{T}_{0}^{\prime}(\tau, f)$ is strictly increasing. Let $\tau_{1}<\tau_{2}$ and note that $R^{\prime} \backslash c_{\mathcal{T}_{0}^{\prime}\left(\tau_{2}, f\right)}^{\prime}$ decomposes into two disjoint open sets $\Pi_{\mathcal{T}_{0}^{\prime}\left(\tau_{2}, f\right)}^{\prime}=M^{\prime} \cup\left\{z^{\prime} \in R^{\prime} \backslash M^{\prime}: t^{\prime}\left(z^{\prime}\right)<\right.$ $\left.\mathcal{T}_{0}^{\prime}\left(\tau_{2}, f\right)\right\}$ and $\left\{z^{\prime} \in R^{\prime} \backslash M^{\prime}: t^{\prime}\left(z^{\prime}\right)>\mathcal{T}_{0}^{\prime}\left(\tau_{2}, f\right)\right\}$. Since $\Pi_{\mathcal{T}_{0}^{\prime}\left(\tau_{2}, f\right)}^{\prime} \supset f \Pi_{\tau_{2}} \supset f c_{\tau_{1}}$ and $c_{\mathcal{T}_{0}^{\prime}\left(\tau_{1}, f\right)}^{\prime} \cap f c_{\tau_{1}} \neq \emptyset$ it follows that $\mathcal{T}_{0}^{\prime}\left(\tau_{1}, f\right)<\mathcal{T}_{0}^{\prime}\left(\tau_{2}, f\right)$.

The proof for $\tau_{0}^{\prime}(\tau, f)$ is similar.

Pro of of (2.2). By using (2.1) and Grötzsch's inequalities we can write

$$
\begin{aligned}
a^{-1}\left[\mathcal{T}_{0}^{\prime}\left(\tau_{2}, f\right)-\tau_{0}^{\prime}\left(\tau_{1}, f\right)\right] & =\operatorname{Mod} \Pi_{\tau_{0}^{\prime}(\tau, f) \mathcal{T}_{0}^{\prime}\left(\tau_{2}, f\right)} \geq \operatorname{Mod} f c_{\tau_{1} \tau_{2}} \\
& \geq K^{-1} \operatorname{Mod} c_{\tau_{1} \tau_{2}}=K^{-1} a^{-1}\left(\tau_{2}-\tau_{1}\right)
\end{aligned}
$$


Further, if $\tau_{0}^{\prime}\left(\tau_{2}, f\right)-\mathcal{T}_{0}^{\prime}\left(\tau_{1}, f\right) \leq 0$, the left hand side of $(2.2)$ is trivial. Suppose that $\tau_{0}^{\prime}\left(\tau_{2}, f\right)-\mathcal{T}_{0}^{\prime}\left(\tau_{1}, f\right)>0$. Then the family $c_{\mathcal{T}_{0}^{\prime}\left(\tau_{1}, f\right) \tau_{0}^{\prime}\left(\tau_{2}, f\right)}^{\prime}$ separates $f c_{\tau_{1}}$ from $f c_{\tau_{2}}$ hence its modulus

$$
a^{-1}\left(\tau_{0}^{\prime}\left(\tau_{2}, f\right)-\mathcal{T}_{0}^{\prime}\left(\tau_{1}, f\right)\right) \leq \operatorname{Mod} f \Pi_{\tau_{1} \tau_{2}} \leq K \operatorname{Mod} \Pi_{\tau_{1} \tau_{2}}=K a^{-1}\left(\tau_{2}-\tau_{1}\right) .
$$

As in [6] and [7] one can discuss equality cases in (2.2) which appear when $f c_{\tau}=$ $c_{\tau^{\prime}}^{\prime}$ and the characteristic ellipses of $f$ have a special orientation (are tangent or orthogonal) with respect to the level lines.

2.3. Now we shall consider the family $\mathcal{F}$ of all $K$-qc homeomorphisms $f: R \rightarrow$ $R^{\prime}$ with $f(M)=M^{\prime}$, and, by adding in the case $R=R^{\prime}=\mathbb{C}$ the hypothesis that $M$ contains at least two points, we obtain inequalities valid for the whole family. To this end we define

$$
\tau_{0}^{\prime}(\tau)=\inf \left\{\tau_{0}^{\prime}(\tau, f): f \in \mathcal{F}\right\} \quad \text { and } \quad \mathcal{T}_{0}^{\prime}(\tau)=\sup \left\{\mathcal{T}_{0}^{\prime}(\tau, f): f \in \mathcal{F}\right\} .
$$

Proposition 2.1. There are extremal functions $f_{0 \tau}$ and $F_{0 \tau}$ in $\mathcal{F}$ such that

$$
\tau_{0}^{\prime}(\tau)=\tau_{0}^{\prime}\left(\tau, f_{0 \tau}\right) \quad \text { and } \quad \mathcal{T}_{0}^{\prime}(\tau)=\mathcal{T}_{0}^{\prime}\left(\tau, F_{0 \tau}\right)
$$

Proof of the existence of $f_{0 \tau}$. By the definition of $\tau_{0}^{\prime}(\tau)$ there exists a sequence $\left\{f_{n}\right\} \subset \mathcal{F}$ such that $\tau_{0}^{\prime}\left(\tau, f_{n}\right) \rightarrow \tau_{0}^{\prime}(\tau)$ as $n \rightarrow \infty$. Theorem 1.0 (or Remark 1.2 for $\mathbb{C}$ ) implies that there is a subsequence of $\left\{f_{n}\right\}$, denoted again by $\left\{f_{n}\right\}$, which l.u. converges to $f_{0} \in \mathcal{F}$. Take a sequence $\left\{z_{n}\right\} \subset c_{\tau}$ such that for $z_{n}^{\prime}=f_{n}\left(z_{n}\right)$ we have $t^{\prime}\left(z_{n}^{\prime}\right)=\tau_{0}^{\prime}\left(\tau, f_{n}\right)$. Selecting a subsequence from $\left\{z_{n}\right\}$, denoted again by $\left\{z_{n}\right\}$, we can suppose that $z_{n} \rightarrow z^{*} \in c_{\tau}$. One easily verifies that $z_{n}^{\prime} \rightarrow f_{0}\left(z^{*}\right):=z^{* \prime}$. Thus $t^{\prime}\left(z_{n}^{\prime}\right) \rightarrow t^{\prime}\left(z^{* \prime}\right)$, i.e. $\tau_{0}^{\prime}(\tau)=t^{\prime}\left(z^{* \prime}\right) \geq \tau_{0}^{\prime}\left(\tau, f_{0}\right)$, and by definition of $\tau_{0}^{\prime}(\tau)$ this implies $\tau_{0}^{\prime}(\tau)=\tau_{0}^{\prime}\left(\tau, f_{0}\right)$, i.e. $f_{0 \tau}:=f_{0}$.

The proof for $F_{0 \tau}$ is similar.

THEOREM 2.2. The functions $\tau_{0}^{\prime}$ and $\mathcal{T}_{0}^{\prime}$ are strictly increasing and satisfy for $\tau_{1}<\tau_{2}$ the inequalities

$$
K^{-1}\left[\tau_{0}^{\prime}\left(\tau_{2}\right)-\mathcal{T}_{0}^{\prime}\left(\tau_{1}\right)\right] \leq \tau_{2}-\tau_{1} \leq K\left[\mathcal{T}_{0}^{\prime}\left(\tau_{2}\right)-\tau_{0}^{\prime}\left(\tau_{1}\right)\right]
$$

Proof. If $\tau_{1}<\tau_{2}$ then $\mathcal{T}_{0}^{\prime}\left(\tau_{1}\right)=\mathcal{T}_{0}^{\prime}\left(\tau_{1}, F_{0 \tau_{1}}\right)<\mathcal{T}_{0}^{\prime}\left(\tau_{2}, F_{0 \tau_{1}}\right) \leq \mathcal{T}_{0}^{\prime}\left(\tau_{2}\right)$.

Further, (2.3) follows from (2.2) taking into account that for every $\tau$ and every $f \in \mathcal{F}, \tau_{0}^{\prime}(\tau) \leq \tau_{0}^{\prime}(\tau, f)$ and $\mathcal{T}_{0}^{\prime}(\tau) \geq \mathcal{T}_{0}^{\prime}(\tau, f)$.

\subsection{Examples of functions $t$}

2.4.1. Sario capacity function ([15], [16], Ch. V, [17], p. 179, [18], Ch. III). Let $R$ be an open Riemann surface, $z_{0}$ a point in $R, z$ a l. parameter in a neighbourhood $v$ of $z_{0}$. The Sario capacity function $p_{R}\left(, z_{0}\right)$ of $R$ (or of its ideal boundary $\Gamma$ ) with respect to $z_{0}$ and the 1 . parameter $z$ is defined by the properties:

(i) $p_{R}\left(, z_{0}\right)$ is harmonic on $R \backslash\left\{z_{0}\right\}$.

(ii) $p_{R}\left(z, z_{0}\right)=\log \left|z-z_{0}\right|+h(z)$ in $v$, where $h$ is harmonic and $h\left(z_{0}\right)=0$. 
(iii) In the family of functions $\{\varphi\}$ on $R$ with the properties (i) and (ii), $p_{R}\left(, z_{0}\right)$ minimizes the integral $\int_{\Gamma} \varphi * d \varphi:=\lim _{n \rightarrow \infty} \int_{\Gamma_{n}} \varphi * d \varphi$, where $\Gamma_{n}=\partial \Pi_{n}, \Pi_{n}$ is a regular region of $R$, the sequence $\left\{\Pi_{n}\right\}$ forms an exhaustion of $R$ and $\Pi_{0} \ni z_{0}$. The integral $\int_{\Gamma} p_{R} * d p_{R}=k_{\Gamma}$ is the Robin constant of $\Gamma$ with respect to $z_{0}$ and $z$, and $c_{\Gamma}=e^{-k_{\Gamma}}$ the capacity of $\Gamma$. We have $k_{\Gamma}=\infty$ or $k_{\Gamma}<\infty$ according as $R$ is of parabolic or hyperbolic type.

The class $R_{p}$ of open Riemann surfaces for which the level lines $c_{\tau}=\{z \in R$ : $\left.p_{R}\left(z, z_{0}\right)=\tau\right\}$ are compact has been studied by M. Nakai [13], L. Sario and K. Noshiro [17], p. 30, Ch. IV, §1, B. Rodin and L. Sario [14], p. 231, L. Sario and M. Nakai [16], Ch. V, §3, 13B. By means of the Evans-Selberg potential, M. Nakai proved that $R_{p}>O_{G}$, but $R_{p}$ also contains hyperbolic surfaces, e.g. all surfaces which are the interior of a compact bordered Riemann surface.

Thus if we take $R \in R_{p}$ and $M=\left\{z_{0}\right\}, p_{R}\left(, z_{0}\right)$ gives an example of a function $t$ with $\tau_{0}=-\infty, \mathcal{T}_{0}=k_{\Gamma}, a=2 \pi$, and Theorems 2.1 and 2.2 apply for $R, R^{\prime} \in R_{p}, M=\left\{z_{0}\right\}, M^{\prime}=\left\{z_{0}^{\prime}\right\}$ (except for Theorem 2.2 when $R=R^{\prime}=\mathbb{C}$ and we have to choose a capacity function with two logarithmic poles). In [6], beside the case of the capacity function, we treated the related case of the Green function, of course for hyperbolic surfaces.

2.4.2. Jurchescu modulus function ([9], [10], [18], Ch. IV). Let $M$ be the closure of a regular region $\Pi_{0}$ on the open Riemann surface $R$ and $\Gamma_{0}=\partial \Pi_{0}$. The modulus function of $\Gamma$ with respect to $\Gamma_{0}$ is a continuous function $u_{R}\left(, \Gamma_{0}\right)=$ $u_{\Gamma}\left(, \Gamma_{0}\right): R \backslash \Pi_{0} \rightarrow \mathbb{R}$ with the properties:

(i) $u_{R}$ is harmonic in $R \backslash \mathrm{cl} \Pi_{0}$ and $u_{R}=0$ on $\Gamma_{0}$.

(ii) $\int_{\Gamma_{0}} * d u_{R}=1$.

(iii) $u_{R}$ minimizes the Dirichlet integral $D(\varphi)=\iint_{R \backslash \mathrm{cl} \Pi_{0}}|\operatorname{grad} \varphi|^{2} d x d y$ in the family of functions $\{\varphi\}$ with the properties (i)-(ii).

The modulus function $u_{R}\left(, \Gamma_{0}\right)$ gives another example of a function $t$, again for the class $R_{p}$ of Riemann surfaces, since this class may also be characterized by the compactness of the level lines of $u_{R}\left(, \Gamma_{0}\right)$, independently of $\Gamma_{0}$ (see [3]). In this case $\tau_{0}=0, \mathcal{T}_{0}=\mu_{\Gamma}$, the modulus of $\Gamma$ with respect to $\Gamma_{0}$, which is finite or infinite according as $R$ is hyperbolic or parabolic. Here again Theorems 2.1 and 2.2 apply for $R, R^{\prime} \in R_{p}, M=\operatorname{cl} \Pi_{0}, M^{\prime}=\operatorname{cl} \Pi_{0}^{\prime}$. We studied this case in [7], where we also consider the harmonic measure of $\Gamma$ with respect to $\Gamma_{0}$ in the hyperbolic case.

2.4.3. Other examples of functions $t$ to which the above results may be applied are given by the capacity function or the modulus function of a part $\beta \subset \Gamma$, in particular of an element of $\Gamma$.

3. An extension of Gehring's Theorem. By applying the distortion theorems in $\S 2$ to the capacity function 2.4.1, we can establish the following extension of Gehring's Theorem [4]. 
TheOREm 3.1. Let $R$ and $R^{\prime}$ be Riemann surfaces of class $R_{p}$ which are not conformally equivalent to $\mathbb{C}, z_{0} \in R, z_{0}^{\prime} \in R^{\prime}$ and suppose that the family $\mathcal{F}$ of $K$-qc homeomorphisms $f: R \rightarrow R^{\prime}$ with $f\left(z_{0}\right)=z_{0}^{\prime}$ is non-void. Let $z_{j}, j=1,2$, be two distinct points in $R \backslash\left\{z_{0}\right\}$ and for a homeomorphism $f \in \mathcal{F}$ define $z_{j}^{\prime}=f\left(z_{j}\right)$, $\tau_{j}=p_{R}\left(z_{j}, z_{0}\right)$ and $\tau_{j}^{\prime}=p_{R^{\prime}}\left(z_{j}^{\prime}, z_{0}^{\prime}\right)$. If $m \leq \tau_{1} \leq \tau_{2} \leq M, m, M \in \mathbb{R}, M \leq k_{\Gamma}$, then

$$
\tau_{1}^{\prime} \leq \tau_{2}^{\prime}+K C
$$

with a positive constant $C$ given by

$$
C=\mathcal{T}_{0} \circ \mathcal{T}_{0}^{\prime}(M)-\tau_{0} \circ \tau_{0}^{\prime}(m)
$$

where $\tau_{0}^{\prime}$ and $\mathcal{T}_{0}^{\prime}$ are the functions defined in 2.3 while $\tau_{0}$ and $\mathcal{T}_{0}$ correspond to the inverse homeomorphisms: $\tau_{0}\left(\tau^{\prime}\right)=\inf \left\{\tau_{0}\left(\tau^{\prime}, f^{-1}\right): f \in \mathcal{F}\right\}, \tau_{0}\left(\tau^{\prime}, f^{-1}\right)=$ $\min \left\{p_{R}\left(f^{-1}\left(z^{\prime}\right), z_{0}\right): z^{\prime} \in c_{\tau^{\prime}}^{\prime}\right\}$ and similiarly for $\mathcal{T}_{0}\left(\tau^{\prime}\right), \mathcal{T}_{0}\left(\tau^{\prime}, f^{-1}\right)$.

Proof. If $\tau_{1}^{\prime} \leq \tau_{2}^{\prime}$, the inequality (3.1) is trivial. Suppose $\tau_{1}^{\prime}>\tau_{2}^{\prime}$. Theorem 2.2 applied to the family of the inverse homeomorphisms $f^{-1}$ with $f \in \mathcal{F}$ implies $\tau_{1}^{\prime}-\tau_{2}^{\prime} \leq K\left[\mathcal{T}_{0}\left(\tau_{1}^{\prime}\right)-\tau_{0}\left(\tau_{2}^{\prime}\right)\right]$. Further, from the definitions and monotonicity properties, $\tau_{1}^{\prime} \leq \mathcal{T}_{0}^{\prime}\left(\tau_{1}\right) \leq \mathcal{T}_{0}^{\prime}(M)$ and $\tau_{2}^{\prime} \geq \tau_{0}^{\prime}\left(\tau_{2}\right) \geq \tau_{0}^{\prime}(m)$, so that $\mathcal{T}_{0}\left(\tau_{1}^{\prime}\right) \leq$ $\mathcal{T}_{0} \circ \mathcal{T}_{0}^{\prime}(M)$ and $\tau_{0}\left(\tau_{2}^{\prime}\right) \geq \tau_{0} \circ \tau_{0}^{\prime}(m)$.

Rem ark 3.1. Theorem 3.1 also applies to compact Riemann surfaces $S$ and $S^{\prime}$ which are not conformally equivalent to $\widehat{\mathbb{C}}$, by choosing two points $z_{\infty} \in S$, $z_{\infty}^{\prime} \in S^{\prime}$ and considering the family $\mathcal{F}$ of $K$-qc homeomorphisms $f: S \rightarrow S^{\prime}$ with $f\left(z_{h}\right)=z_{h}^{\prime}, h=0, \infty$. We consider the surfaces $R=S \backslash\left\{z_{\infty}\right\}$ and $R^{\prime}=S^{\prime} \backslash\left\{z_{\infty}^{\prime}\right\}$ of class $R_{p}$, the corresponding capacity functions $p_{R}\left(, z_{0}\right)$ and $p_{R}\left(, z_{0}^{\prime}\right)$, and if $m \leq \tau_{1} \leq \tau_{2} \leq M$ we obtain again (3.1) with (3.2).

\section{References}

[1] L. V. Ahlfors and L. Sario, Riemann Surfaces, Princeton Univ. Press, Princeton, N.J., 1960.

[2] C. Andreian Cazacu, Sur un problème de L. I. Volkovyski, Rev. Roumaine Math. Pures Appl. 10 (1965), 43-63.

[3] - On the class $R_{p}$ of Riemann surfaces, communicated at a meeting of the Polytechnical Inst. Chişinau (1993), to appear.

[4] C. Andreian Cazacu and V. Stanciu, On a Gehring's Theorem, to appear in the volume dedicated to the centenary of Acad. N. Muskhelishvili by the Georgian Academy of Sciences.

[5] —, - Normal families of quasiconformal homeomorphisms I, II, An. Univ. Bucureşti Mat. 40 (1991), 7-14 and 41 (1992), 3-6.

[6] - Distortion of the level lines of the capacity functions under $K$-qc mappings, Studia Univ. Babeş-Bolyai Math. 36 (2) (1991), 51-61.

[7] - Distortion of the level lines of the modulus functions under K-qc mappings, Serdica 19 (1993), 113-120.

[8] F. W. Gehring, Characteristic Properties of Quasidisks, Presses Univ. Montréal, 1982. 
[9] M. Jurchescu, Suprafeţe riemanniene cu frontiera absolut discontinuă, Disertaţie, Bucureşti, 1956.

[10] —, Modulus of a boundary component, Pacific J. Math. 8 (1958), 791-804.

[11] O. Lehto, Univalent Functions and Teichmüller Spaces, Springer, New York, 1987.

[12] O. Lehto and K. I. Virtanen, Quasiconformal Mappings in the Plane, Springer, Berlin, 1973.

[13] M. Nakai, On Evans potential, Proc. Japan Acad. 38 (1962), 624-629.

[14] B. Rodin and L. Sario, Principal Functions, Van Nostrand, Princeton, N.J., 1968.

[15] L. Sario, Capacity of the boundary and of a boundary component, Ann. of Math. 59 (1954), 135-144.

[16] L. Sario and M. Nakai, Classification Theory of Riemann Surfaces, Springer, Berlin, 1970.

[17] L. Sario and M. Noshiro, Value Distribution Theory, Van Nostrand, Princeton, N.J., 1966.

[18] L. Sario and K. Oikawa, Capacity Functions, Springer, New York, 1969. 